Témoigner Témoigner. Entre histoire et mémoire

Getuigen Revue pluridisciplinaire de la Fondation Auschwitz

$123 \mid 2016$

Traduire le témoignage

\title{
Traduire la révolution égyptienne
}

Traduction, témoignage et activisme : entretien avec Samah Selim

Translating the Egyptian Revolution. Translation, Testimony, Activism:

An Interview with Samah Selim

Tom Toremans et Samah Selim

(2) OpenEdition

Journals

Édition électronique

URL : https://journals.openedition.org/temoigner/5414

DOI : 10.4000/temoigner.5414

ISSN : 2506-6390

Éditeur :

Éditions du Centre d'études et de documentation Mémoire d'Auschwitz, Éditions Kimé

Édition imprimée

Date de publication : 1 octobre 2016

Pagination : 143-151

ISBN : 987 2-9600926-4-6

ISSN : 2031-4183

Référence électronique

Tom Toremans et Samah Selim, «Traduire la révolution égyptienne», Témoigner. Entre histoire et mémoire [En ligne], 123 | 2016, mis en ligne le 02 novembre 2021, consulté le 04 novembre 2021. URL : http://journals.openedition.org/temoigner/5414 ; DOI : https://doi.org/10.4000/temoigner.5414 


\section{Traduire la révolution égyptienne}

\section{Traduction, témoignage et activisme : entretien avec Samah Selim}

\author{
$\rightarrow$ Tom Toremans \\ KU Leuven \\ $\rightarrow$ Samah Selim \\ Rutgers University
}

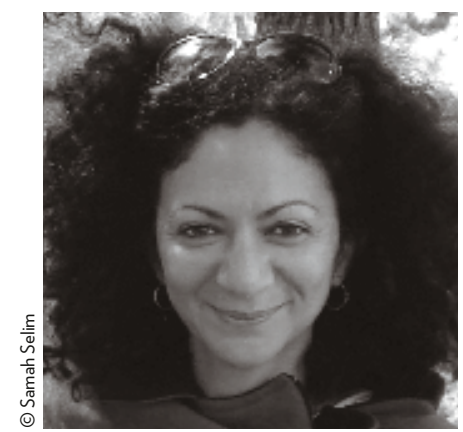

Samah Selim est à la fois universitaire, traductrice et activiste. Elle a produit de nombreux ouvrages tout à fait pertinents dans le cadre de cette publication spéciale sur la relation entre traduction et témoignage. C'est plus particulièrement le tournant pris depuis la recherche universitaire en littérature arabe et en traduction pour une implication activiste plus directe avec la traduction, dans le sillage de la révolution égyptienne en 2011, qui sera mis en lumière. Actuellement professeur à l'Université Rutgers dans le département de langues et littératures d'Afrique, du Moyen-Orient et de l'Asie du Sud-Est, Samah Selim a auparavant enseigné à l'Université de Columbia, de Princeton et d'Aix-en-Provence. Elle est également codirectrice du module de littérature du programme de recherche postdoctoral situé à Berlin, Europe in the Middle East; the Middle East in Europe. Ses recherches publiées concernent principalement la littérature arabe moderne en Égypte et au Proche-Orient, ainsi que la politique de la traduction dans les contextes (post-)coloniaux. En 2004, elle a publié une monographie, The Novel and the Rural Imaginary in Egypt 1880-1995, qui explore la relation entre l'émergence du roman, la politique de la représentation nationaliste et la question paysanne dans l'Égypte du XXe siècle.

Samah Selim a également traduit des ouvrages de Yahya Taher Abdullah, Khaled Ziadeh, Mohamed Makhzangi et Jurji Zaydan. Elle est la première traductrice à avoir remporté à la fois le prix Banipal et le prix de l'Arkansas pour la traduction d'une œuvre littéraire de langue arabe.

En 2012, son implication dans la traduction prend une tournure activiste lorsqu'elle devient membre de l'unité de sous-titrage vidéo de Mosireen, un collectif à but non lucratif (www.mosireen.org). Elle est également membre du « Réseau de recherche Mataroa pour un nouvel imaginaire méditerranéen » (www.mataroanetwork.org). Inspirée par cette implication universitaro-activiste à plusieurs facettes dans la traduction et le témoignage, nous avons eu un entretien par e-mail avec Samah Selim en juillet dernier. 
Traduire la révolution égyptienn
(suite)
Vos travaux les plus récents sur la traduction activiste ont été influencés par votre engagement en tant que sous-titreuse au sein du collectif vidéo Mosireen en 2012-2013. Pouvez-vous nous donner une idée concrète de cet engagement et de son influence sur vos travaux académiques?

Samah Selim : Lorsque Mosireen a lancé son appel aux traducteurs sur les réseaux sociauxà l'automne 2012,je connaissais déjà bien leurs travaux de documentation du soulèvement J'étais a jorsencongés de recherche au Caire, pour un projetd'ouvrege

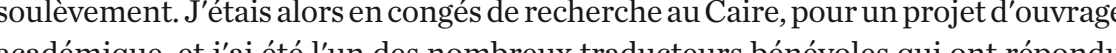
academique, et jai été lun des nombreux traducteurs bénévoles qui ont répondu à cet appel. Tout comme de nombreux Égyptiens a l'etranger, j'ai vécu les début de la révolution dans un espace virtuel. Les premiers mois de 2011 étaient particulièrement stressants, j'étais comme forcée à vivre dans des univers parallèles. Mon premier véritable contact avec la rue a eu lieu au cours de l'été 2011 et, à partir de ce moment-là,j'ai eu la chance de passer la majeure partie des quatre années suivantes au Caire. Ainsi, avant même de commencer à travailler avec Mosireen, j'étais sur le terrain, au cœur de la plupart des événements qui constituent l'ensemble de leurs très nombreuses archives vidéos. Bien évidemment, c'était une période d'agitation, d'effervescence et de violence. Personne ne savait vraiment ce qui pouvait se passe d'un jour à l'autre. Tout d'un coup, il y avait autant d'acteurs politiques sur la scène (a) égyptienne que de persones sur le terrain. Mon travail avec Mosireen faisait parti intégrante de cette situation de chaos géneral qui aspirait à donner du sens et une structure. Je n'exagère vraiment pas lorsque je dis que «travailler» sur autre chose que la révolution elle-même était impossible pour une foule de personnes en Égypte, en particulier pour les écrivains ou les universitaires comme moi. Mon ouvrage s'est retrouvé purement et simplement mis de côté. Il est devenu complètement sans importance, voire même inapproprié, et ce n'est que bien après le coup d'Etat de 2013 que j'ai été en mesure de commencer à vaguement déterminer de quelle manière il pourrait redevenir pertinent. Pendant ces quatre années, l'essentiel de mon travail consistaità traduire (Mosireen mais aussi divers textes issus de la situation politique, tels quel'analyse journastiquetla documentation du Partide l'Alliance poplaire socialiste) ou à effectuer des interventions publiques.

Maintenant, toutes ces années après, je pense pouvoir affirmer que, par rapport à ma profession universitaire et à ma vocation en tant que traductrice, la révolution m'a permis de miversitaire et a ma vocation en tant que traductrice, láration, très largement défini, façonne la base de la culture arabe moderne, y compris la littérature bien évidemment. Mes intérêts dans la traduction ont alors pris de nouveaux chemins. Je ne considère plus autant la traduction en tant que «marché» ou «pont», mais je l'envisage désormais davantage comme une forme de production de savoir radicale.C'estce que reflète mon projet de traduction d'ouvrage actuel (le premier en

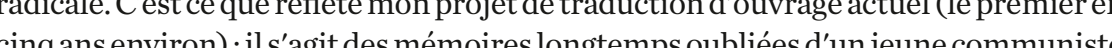
cinptien (uipte années 1970. Ces l'économie politique de la traduction vers l'arabe. L'année dernière, un petit groupe de confrères égyptiens et moi-même avons fondé un collectif pour développer une communauté de traducteurs en ligne afin d'expérimenter de nouvelles façons de penser et pratiquer la traduction en arabe.

Dans votre contribution au récent volume Translating Dissent (Baker, 2016), vous commentez votre engagement avec Mosireen en notant « une continuité remarquable quasiment directe, entrelásésence du témoinna et le travail de traduction (p. 82-83) quasime Pouvez-Vus nus en dire plus sur cette continuite ? La traduction est-elle un processus qui permet la dissémination internationale et interlinguistique des témoignages, ou y
a-t-il une plus grande affinité entre ces deux activités?

Traduire au milieu d'une bataille constitue certainement une façon de disséminer le témoignage, conme vous dites, de tèmoigner (en tant que traducteur et partisan). Tout au moins, cela rend troubles les eaux de ce que les gens appellent $l^{\prime}$ « objectivité». Je n'arrive pas vraiment à imaginer comment un traducteur ou un interprète peut élaborer une pratique «objective» au milieu d'un massacre (par opposition, peut-être, au fait d'être assis dans un bureau ailleurs dans le monde).

$$
\begin{aligned}
& \text {-Affrontements entre la } \\
& \text { police anti-émeute et les } \\
& \text { manifestants rue Mohamed }
\end{aligned}
$$
Mahmoud.

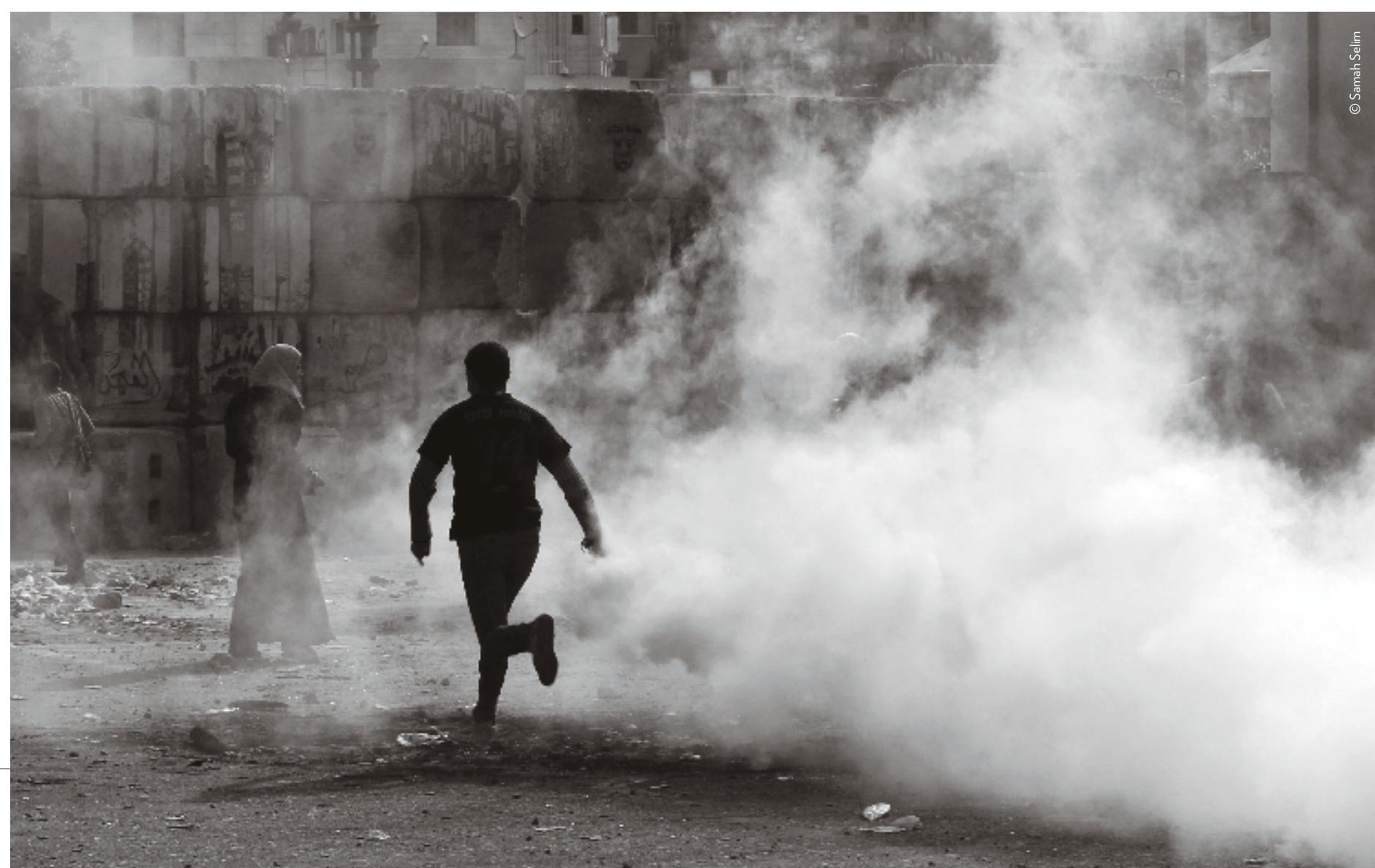


Traduire la révolution égyptienn
(suite)

(1) En 2011, la WRL (War York a commencé à mettre en ouurre une campagne
pour interdire '́utilisation pour interdire ' 'utilisation de
gaz lacrymogenes contre les
mouvements de contestation populaires dans le pays et ailleurs. En juin 2012 , le mouvement toujours en cours, Facing Tear Gas (http:///facingteargas.org/,
et a commencé a recueillir des récits personnels d'hommes et de des gaz lacrymogènes dans ces des gaz lacrymogènes dans ces
différents lieux d'insurrection. L
WRL s'associe à des activistes WRL s'associe à aes activistes
de pays comme 'E'Eypte, le Cour boycotter l'exportation de materiel de contrôle des émeutes
fabriqué aux Ettats-Unis.
Je n'arrive pas à imaginer pourquoi une traductrice s'immiscerait tout d'abord au milieu d'un massacre, sauf à vouloir «témoigner» d'une certaine façon. Parmi les milliers de personnes présentes lors des grands affrontements de 2011 ou 2012 qui ont eu lieu dans la rue, toutes n'étaient pas activement impliquées dans la confrontation avec la police. Beaucoup étaient là pour apporter leur soutien aux combattants (malgré le gaz neurotoxique et le risque de snipers) de différentes façons : premiers secours, approvisionnement en eau et nourriture, chants de guerre ou témoignage. Ce témoignage fait partie de l'arsenal de la révolution, car il constitue la base de la Ce témoignage fait connaissance du collectif des insurgés. Il est très dangereux, et c'est pour cela que l'Etat fera tout ce qui est en son pouvoir pour le supprimer (comme cela est en train
de se produire).

Les archives de Mosireen représentent une forme de ce témoignage et mon travail de traduction quasiment en temps réel faisait partie intégrante du processus de dissémination, à la différence qu'il était à plus grande échelle. Lorsque je travaillais dans la situation de panique que je décris dans l'essai que vous mentionnez, je gardais toujours à l'esprit la conviction que mes amis et camarades à New York, don nombre d'entre eux étaient directement impliqués dans le mouvement Occupy, nous rejoindraient bientôt de façon importante, en regardant les vidéos que nous nouśćch téléchargions quelques heures seulement après les evenements (et parfois même pour élargir le cercle du «nous» des vidéos, et par conséquent le rendre plus fort.

De temps en temps, je retourne sur le site Web de Mosireen, juste pour m'assurer qu'il est encore là, mais aussi pour regarder à nouveau certaines vidéos, et je suis surprise de constater tout ce que j'ai oublié. Bien qu'il soit maintenant très douloureux de les regarder encore une fois, cela reste incroyablement émouvant inspirant, même. Je suis tellement heureuse d'avoir pris part à ce travail qui permet de les garder en vie dans d'autres lieux et d'autres langues.

Lorsque vous mentionnez la politique radicale de la lecture d'Edward Said, vous posez une notion tout aussi politique de la traduction comme « une forme de pièce d'un ensemble » (p. 80) dans laquelle le traducteur se démarque pour "changer le monde, plutôt que de simplement le sonder. " (p. 82) Au cœur de ce concept activiste de la traduction se trouve « une forme d'engagement non seulement envers des mots et des significations, mais également envers des lieux spécifiques porteurs d'histoires politiques, culturelles et sémantiques. » (p. 78) Aussi, vous affirmez que «l'engagement du traducteur envers le lieu de lutte implique la responsabilité de l'obtention de ce contexte lourd et de son rendu lisible auprès des mouvements et communautés d'insurgés à l'étranger.» (p. 85-86) Cette dernière activité que vous qualifiez de « traduction profonde „, qui vise à élir des rése de solidarté internationaux. Ce mode de traduction est qui vise a ét à une « circulation constante de l'image et du spectacle sur les réseaux sociaux » (p. 84).

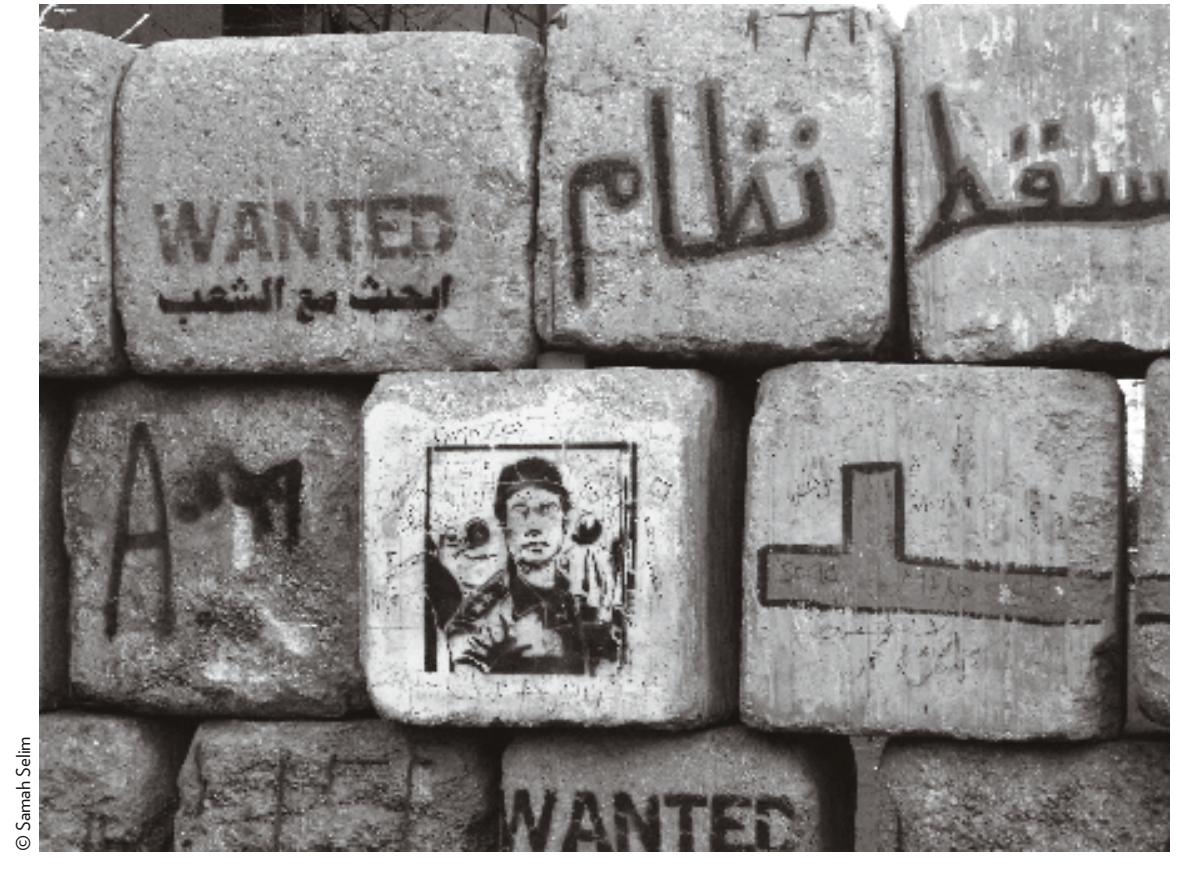

-Cloison de séparation près du Ministere de l'Intérieur. photo d'un agent de police tristement célèbre pour avoir visé les yeux des manifestants durant les protestations circulait sur les média sociaux.

Comment concevez-vous la relation entre ces deux modes de traduction, l'un étroitement lié à des lieux et des événements locaux, l'autre cherchant à connecter une communauté internationale à l'étranger? Cela fait-il partie de votre travail en tant que traductrice, sous-titreuse et universitaire?

Je pense avoir probablement déjà répondu, en partie, à cette question dans ma réponse précédente, mais pour résumer, mavisualisation de ces deux modes est spa tiale, voire mêmea , mistectura throp vire le propag rempleur, si les cercles de résonace et de possibilté sont éphémères. La traduction profonde, quant à elle, consiste plutôtà creuser età bâtir, à modeler l'espace et à créer une structure. Elle cherche à capturer la résonance en cours d'atténuation de la crise et à en faire un savoir, relatif, autant que possible, au contexte profond de la crise : son histoire, ses bases économicopolitiques, son impact culturel, mais aussi les moindres détails, ainsi que les tenants et aboutissants des événements quotidiens qui l'entourent. La traduction profonde constitue la base du type de compréhension politique sans frontières qui permet à la solidarité de fonctionner et qui rend l'activisme local possible et efficace La campagne de la WRL contre les gaz lacrymogènes ${ }^{1}$ dont je parle dan

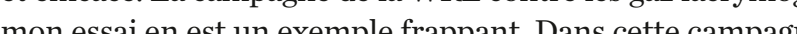
étroit collo en étroite collaboration avec le personnel des douanes égyptiennes qui avait bloqué le 
collaboration a abouti à une campagne à grande échelle contre le fabricant d'armes américain Combined Systems, et la documentation principale (documents, rapports campagnes d'information) était régulièrement traduite d'une langue à l'autre.

Un autre exemple : les images et séquences provenant du Caire en 2011 et 2012 n'étaient diffusées aux États-Unis que dans leurs grandes lignes (contestataires et police), même dans les cercles d'activistes. Seule une forme de traduction profonde aurait permis de mettre en avantl'agitationdu moment, avec ses nombreux acteurs (exteurs et ses inters les pour les personnes œuvrant dans des contextes d'insurrection similaires à l'étranger. Tout un éventail de partis politiques s'est engouffré dans la politique électorale entachée de sang qui a commencé en 2011. À ce moment-là, il semblait que, pour la première fois depuis des années, il y avait une véritable chance de voir émerger une gauche démocratique radicale et organisée. Toute personne de la gauche internationale sérieusement intéressée par des alliances au niveau mondial, ou même effectuant juste une analyse approfondie de la révolution en Égypte, aurait nécessairement eu besoin de traductions. J'ai traduit la plate-forme récemment créée du Parti de l'Alliance populaire socialiste en 2011, juste après que l'encre ait fini de sécher sur celle-ci. Ceci n'était que le commencement de ce qui,je l'espérais, serait un véritable site Web en anglais (hólas, le parti s'est ignoblement efrón effondré l'ordre du jour. En raison du peu de traductions profondes de ce genre durant la révolution, très peu d'alliés potentiels a l'etranger savaient ce qui se jouait et pourquoi, lorsque 2013 est arrivé. Bien évidemment, mon propos n'est absolument pas d'insinuer que le type approprié de travaux de traduction aurait sauvé la révolution égyptienne, mais uniquement que la création d'une alliance internationale engagée est pratiquement impossible sans celui-ci.

D'une certaine façon, tout ceci me amèneà 2016. Je crois que c'estcette envie impérieuse de réaliser cst cette (a) ductions «profondes» qui m'a amenee a travailler sur l'ouvrage d'Arwa Saleh, The Stillborn: A young woman's notes from the student movement generation in Egypt, que j'ai mentionné précédemment. L'ouvrage est si profondément façonné par une histoire locale «non autorisée», et il s'adresse si directement à une audience locale (et très particulière de surcrôt, à savoir les cam de surcroit, à savoir les camarades de Saleh, anciens étudiants commu-
nistes des années 70), que lui donner un sens en anglais, y compris pour les «parties intéressées», est une tâche vraiment ardue. Pourtant, je suis persuadée que cet ouvrage aide grandement le lecteur cible anglophone, quelle que soit son origine, à comprendre quelque chose d'essentiel concernant l'origine de la révolution de 2011 et pourquoi elle a «échoué», à supposer qu'elle ait échoué. Ce sont des questions que nous nous sommes posées, de très nombreux Égyptiens et moi-même, au cours de ces dernières années, mais je pense aussi qu'elles doivent forcément susciter l'intérêt de toute personne qui s'intéresse au passé, au présent et à l'avenir de la gauche internationale.

Votre article se termine par un appel à un « effort collectif organisé» (p. 86), basé sur les récits des communautés (en référence aux récents travaux de Mona Baker, 2010). sur les récits des communautés (en référence aux récents travaux de Mona Baker, 2010).
Constatez-vous des cas concrets de traduction activiste aujourd'hui, par exemple dans le contexte des actuels conflits/révolutions au Moyen-Orient ? Aussi, cette traduction activiste permet-elle l'émergence de témoignages « sous embargo » au-delà de la forme dominante, au niveau international, de la couverture médiatique « fatalement empreinte de paradigmes néo-libéraux et eurocentriques » ( $p .86)$ ?

Oui, il existe certainement de nombreux projets d'information activiste sur le Web dont la traduction constitue une composante majeure : par exemple, les comWeb dont la trad munautés deríne Real Internatioal Neus Netion), Anarchist Network également un site Web dédie). Pour ces deux derniers, en particulier, le MoyenOrient est au cour de leurs centres d'interêt, et ils présentent des traductions entre et vers l'arabe, le persan, le kurde, l'anglais, l'espagnol et le français. Ils constituent des forums pour l'analyse alternative et la dissémination des informations, en grande partie par le biais de mécanismes de traduction de différentes sortes. Ces groupes ont tendance à être essentiellement basés dans des collectifs anarchistes partout dans le monde, mais ils n'intègrent pas forcément une politique de traduction structurée, et ne présentent pas non plus une identité corporative en tant que traducteurs militants, contrairement à Tlaxcala ou Babels Alors que Tlaxcala est une chaurs d'échange dis mission déclarée met explicitement au premier plan le travail du traducteur comme étant absolument central pour la production de connaissances alternatives et l'établissement d'une solidarité internationale. Babels, de son côté, se compose d'un corps professionnel de traducteurs/interprètres bénévoles pour le Forum social mondial. Pour autant, tous ces groupes ont en commun leur compréhension horizontale, bénévole et plutôt radicale de l'importante de la traduction dans la lutte internationaliste à l'ère des médias néo-libéraux et institutionnels. Et je suis convaincue que, plus la mainmise de ces médias institutionnels mondiaux va s'étendre, plus des groupes comme ceux-ci vont continuer à proliférer, évoluer et s'épanouir.

Hormis une spécialisation dans la littérature égyptienne et arabe, vous êtes également une traductrice primée des travaux d'Yahya Taher Abdullah et de Jurji Zaydan, 
entre autres. Comment reliez-vous ce travail de traduction à votre engagement en tant qu'activiste? Et envisageriez-vous la traduction littéraire également comme une forme de témoignage ou d'attestation?

C'est une question plutôt difficile, notamment parce que j'ai ressenti l'existence d'un «avant» et d'un «après» 2011 dans mon travail de traduction, à laquelle je n'ai pas encore suffisamment réfléchi. Ce dont je suis sûre, c'est que la traduction littéraire ne porte en aucune façon le caractère immédiat et urgent du travail tion littérion nu traval de traduction politique. La traduction litteraire s'attache à reproduire le ton, le style et le rythme dans la langue cible. C est un travail plus lent, plus personne et contemplatif, qui ouvre un autre type de relation avec le public cible. En outre l'infrastructure de publication et de distribution de la traduction littéraire, ou tout du moins de la traduction littéraire que j'ai effectuée jusqu'à présent, est également très différente du type de traduction politique dont nous avons discuté. Que cela vous plaise ou non, vous travaillez au sein d'une structure commerciale, et votre lectorat peut avoir des types d'intérêts et de préoccupations très différents des vôtres. Selon que vous aurez choisi ou non les textes à traduire, votre propre relation au texte peut varier.

Le plus souvent, lorsque je décide que je veux traduire un texte littéraire, c'est en raison de sa beauté, de son exaltation et de sa puissance; c'est parce que je suis convaincue qu'il peut apporter du plaisir et de la connaissance au lecteur anglophone, car il peut lui offrir une petite lucarne dans un monde d'affects et de significations vaste et complexe, à la fois étrange et familier. Cette dialectique est ce qu crée la possibilité de tout type d'implication, de défi et de reconnaissance, au sein des cultures et entre elles. Pour moi, ce processus, cette relation est distinct(e) de la traduction politique, mais en même temps, cette dernière expérience (The Stillborn) est peut-être un mélange des deux, dans lequel la lenteur et la recherche de la tonalité du litteraire rejoint à un certain niveaul'urgence et le ciblage plus direct du lecteur du politique.

Vous travaillez actuellement sur une monographie relative à la traduction, à la modernité et à la fiction populaire au début du XXe siècle en Égypte. Pouvez-vous nous en nité et à la fiction poput

Cela fait maintenant quelques années que je travaille sur cet ouvrage. Lorsque Jai commencé, l'étude de la Nahda («Renaissance», en arabe moderne) débutait tout juste dans les universités américaines et britanniques. Mon intérêt pour cette période (milieu du XIX ${ }^{e}$ siècle jusqu'au début/milieu du XXe siècle environ) vient de mon intérêt pour les origines du roman en arabe. C'est la période à laquelle le roman émerge en tant que genre littéraire majeur partout dans le monde arabe, mais plus particulièrement en Égypte, qui, durant cette période, était le point de rencontre des écrivains, éditeurs et intellectuels de la région. En fait, c'est ce sujet qui m'a intéressée en tout premier lieu dans la traductologie. La traduction et l'adaptation ont été les voies par lesquelles le roman est arrivé en langue arabe, bien que les récits littéraires nationalistes tendaient à ignorer ce qui est principalement une immense archive désorganisée de fictions adaptées et hautement populaires. L'ouvrage est axé sur un périodique de fiction du début du XXe siècle, publié au Caire entre 1904 et 1911. Dans celui-ci,j'essaie, dans un premier temps, d'explorer la centralité de la traduction et de l'adaptation, pour le genre littéraire qu'est le roman à un niveau global, ainsi qu'au-delà des frontiê (a) de la littérature nationale, pis dans undeuxiente tenps, jo recherche un nouveau mode de pensée sur la culture et le voyage culturel, en opposition à la façon dont ils
tendent à être définis dans les études postcoloniales.

Il devrait sortir en 2018 chez Palgrave, mais je suis encore plus enthousiasmée par sa sortie en arabe peu de temps après. J'ai donné quelques conferences sur celui-ci au Caire en 2015, et j'ai été impressionnée par l'intérêt qu'il a suscité, en particulier parmi les non-universitaires. C'était assez bouleversant d'être directement confrontée à cette soif de nouveaux paradigmes critiques susceptibles d'aider à rompre l'emprise de la politique culturelle établie sur la pensée indépendante (que l'on pourrait appeler urebelle ) concernant la culture et lidentité culturelle. (que lon pon Il y a tellement de choses dans l'histoire des cultures arabes qui font démentir les instruments disciplinaires et ordonnés que sont, d'une certaine manière, tous les régimes théologiques. Parmi les personnes qui m'ont approchée concernant l'ouvrage, beaucoup étaient étonnées de l'existence de l'archive sur laquelle je travaille, de sa portée et de son contexte historique étendu. J'ai même été approchée par une personne de la presse du Caire pour une réédition de certains des romans (au mieux moisissant dans la Bibliothèque nationale, au pire disparaissant rapidement dans les abysses du processus bureaucratique ou le trafic illicite). C'est cette soif qui donne, à mes yeux, de la valeur à mes recherches, ce sentiment de contribuer à un projet intellectuel d'émancipation. 